\title{
Association of "initial CT" findings with mortality in older patients with coronavirus disease 2019 (COVID-19)
}

\author{
Yan $\mathrm{Li}^{1} \cdot$ Zhenlu Yang ${ }^{1} \cdot \mathrm{Tao}^{\mathrm{Ai}}{ }^{1} \cdot$ Shandong $\mathrm{Wu}^{2} \cdot$ Liming Xia $^{1}$ (B) \\ Received: 25 March 2020 / Revised: 30 April 2020 / Accepted: 18 May 2020 / Published online: 10 June 2020 \\ (C) European Society of Radiology 2020
}

\begin{abstract}
Objectives To investigate the association of chest CT findings with mortality in clinical management of older patients. Methods From January 21 to February 14, 2020, 98 older patients ( $\geq 60$ years) who had undergone chest CT scans ("initial CT") on admission were enrolled. Manifestation and CT score were compared between the death group and the survival group. In each group, patients were sub-grouped based on the time interval between symptom onset and the "initial CT" scan: subgroup1 (interval $\leq 5$ days), subgroup2 (interval between 6 and 10 days), and subgroup3 (interval $>10$ days). Adjusted ROC curve after adjustment for age and gender was applied.

Results Consolidations on CT images were more common in the death group $(n=46)$ than in the survival group ( $n=52)(53.2 \%$ vs $32.0 \%, p<0.001)$. For subgroup1 and subgroup2, a higher mean CT score was found for the death group (33.0 \pm 17.1 vs $12.9 \pm 8.7$, $p<0.001 ; 38.8 \pm 12.3$ vs $24.3 \pm 11.9, p=0.002$, respectively) and no significant difference of CT score was identified with respect to subgroup3 $(p=0.144)$. In subgroup1, CT score of 14.5 with a sensitivity of $83.3 \%$ and a specificity of $77.3 \%$ for the prediction of mortality was an optimal cutoff value, with an adjusted AUC of 0.881 . In subgroup2, CT score of 27.5 with a sensitivity of $87.5 \%$ and a specificity of $70.6 \%$ for the prediction of mortality was an optimal cutoff value, with an adjusted AUC of 0.895 .

Conclusions "Initial CT" scores may be useful to speculate prognosis and stratify patients. Severe manifestation on CT at an early stage may indicate poor prognosis for older patients with COVID-19.

Key Points

- Severe manifestation on CT at an early stage may indicate poor prognosis for older patients with COVID-19.

- Radiologists should pay attention to the time interval between symptom onsets and CT scans of patients with COVID-19.

- Consolidations on CT images were more common in death patients than in survival patients.
\end{abstract}

Keywords Coronavirus infections · Tomography $\cdot$ Lung diseases $\cdot$ Mortality

\section{Abbreviations}

COVID-19 Coronavirus disease 2019

ECMO Extracorporeal membrane oxygenation

GGO Ground-glass opacity

RT-PCR Reverse transcription

polymerase chain reaction

Liming Xia

xialiming2017@outlook.com

1 Department of Radiology, Tongii Hospital, Tongji Medical College, Huazhong University of Science and Technology, Wuhan 430030, Hubei, China

2 Department of Radiology, Department of Biomedical Informatics, Department of Bioengineering, Intelligent Systems Program, University of Pittsburgh, Pittsburgh, PA 15213, USA

\section{Introduction}

The novel coronavirus infection initially breaks out in Wuhan, China, and, as of April 9, has spread to more than 200 countries and regions in the whole world. The disease caused by the novel coronavirus (SARS-CoV-2) is now named coronavirus disease 2019 (COVID-19) by the World Health Organization (WHO). As of April 9, 2020, there are accumulative 1,436,198 confirmed cases and 85,522 deaths in the whole world, with an average mortality of about $6.0 \%$ [1], which is lower than that of severe acute respiratory syndrome (SARS) [2] and Middle East respiratory syndrome (MERS) [3]. However, the mortality is extremely high in critically ill patients with COVID-19 (62\%) [4], and older patients with comorbidities are at increased risk of developing critical illness and death [4-6]. The severity of COVID-19 poses great 
strain on critical care resources in hospitals, especially when they are not adequately staffed or resourced [4]. Therefore, stratifying the severity of older patients with or without comorbidities on admission is beneficial to allocate medical resources accordingly.

During the outbreak of COVID-19, in clinical practice, auscultation is limited to identify pulmonary lesions due to the isolation of protective clothing. Although radiation dose $[7,8]$ needs to be considered, chest $\mathrm{CT}$ as reported previously plays an important role in disease diagnosis, monitoring, severity stratification, and evaluation of treatment response in patients with COVID-19 $[9,10]$, which goes beyond the ability of reverse transcription polymerase chain reaction (RTPCR).

In this single-center study, aiming to further identify the value of chest CT in clinical management of COVID-19, we evaluated the association of initial chest $\mathrm{CT}$ findings obtained at admission with severity and clinical outcomes in older patients with COVID-19.

\section{Materials and methods}

\section{Study cohort}

This retrospective single-center study was approved by the Ethics Commission of Tongji Hospital and written informed consent was waived due to emergence of epidemic outbreak. Tongji Hospital is the largest general hospital in Wuhan and has been designated to take care the treatment of severe COVID-19 patients by the Chinese government since late January 2020. We retrospectively reviewed 2237 older $(\geq$ 60 years old) patients with confirmed infection of SARSCoV-2 and admitted to Tongji Hospital between January 21 and February 14, 2020. All patients were confirmed with positive results of RT-PCR in throat swab specimens. Of the 2237 patients, as of March 4, 2020, 212 died of COVID-19, 613 recovered and were discharged from the hospital, and other 1412 patients were still in hospitalization. We finally enrolled 98 patients (46 died patients and 52 survival patients) who had undergone chest $\mathrm{CT}$ scans before admitting to the hospital or within $24 \mathrm{~h}$ of admission (Fig. 1). We defined these CT scans as "initial CT" scans. The 52 survival patients were discharged before March 4, 2020, based on the criterion of the diagnosis and treatment program (trial 7th version) published by the National Health Commission of the People's Republic of China [11]. The clinical characteristics of the 98 older patients were collected from electronic medical records. A CT score was calculated using chest $\mathrm{CT}$ for each patient. According to the prognosis, we divided the 98 patients into a survival group

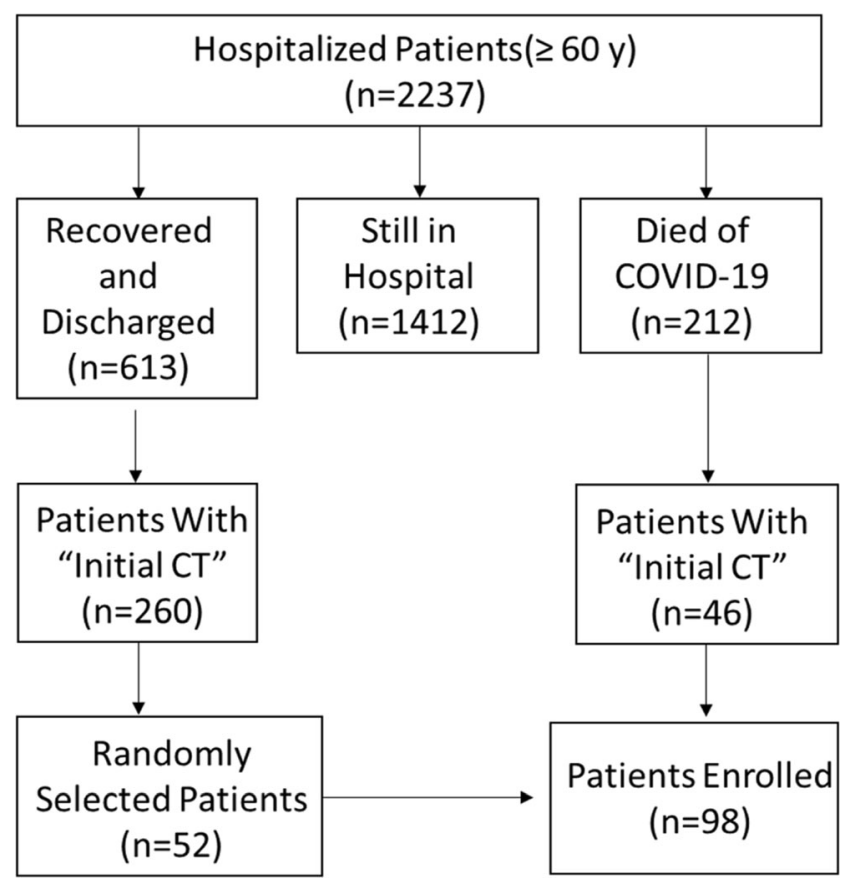

Fig. 1 Flowchart showing the inclusion criteria of this study

and a death group, and the $\mathrm{CT}$ scores were compared between the two groups.

\section{CT technique and image interpretation}

Chest CT images were obtained using the protocol described in a previously reported study [10]. Two certified radiologists (with 8 and 3 years of experience in interpreting thoracic imaging, respectively) were blinded to patient outcomes and reviewed all chest $\mathrm{CT}$ images by consensus.

CT findings were graded on a 3-point scale focused on predominant $\mathrm{CT}$ patterns and based on the classification system as previously described [12]: 1, normal attenuation; 2 , ground-glass opacity (GGO); 3, consolidation. Because this study was focused on evaluating the association between early CT features corresponding to diffuse alveolar damage at early stage and prognosis, Ichikado K's scale points 4, 5, and 6 [12], which are associated with the late proliferative or fibrotic phase, were not used. GGO was defined as increasing density of attenuation that did not conceal the underlying vessels. Consolidation was defined as increasing density of attenuation that concealed vessels.

In addition, the severity of each lung lobe (left upper/lower lobe and right upper/middle/lower lobe) was scored on the basis of predominant lesion extent in the corresponding lobe, as $0(0 \%), 1(1-25 \%), 2(26-50 \%), 3(51-75 \%)$, and $4(76-$ $100 \%)$ [13]. The 4-point scale of severity of lung lobe was multiplied by the 3-point scale of CT findings, generating an 
overall score termed as "CT score." The CT score of each lobe ranged from 0 to 12 , and the finally obtained total cumulative score values of all lung lobes ranged from 0 to 60 .

In the survival $(n=52)$ and death $(n=46)$ groups, the patients were sub-grouped based on the time interval between symptom onset and the "initial CT" scan: subgroup1 (interval $\leq 5$ days), subgroup2 (interval between 6 and 10 days), and subgroup3 (interval $>10$ days).

\section{Statistical analysis}

The software SPSS (IBM SPSS Statistics, version 20.0.0), GraphPad Prism (version 7.00), and R (RStudio, version 1.2.5033) were used for statistical analysis. Continuous variables were expressed as mean \pm standard deviation (SD) or median and interquartile range (IQR). Categorical variables were reported as counts and frequencies in each category. The proportion rates of clinical characteristics in the two groups were compared by the chi-square test.

The mean CT scores of the "initial CT" on admission between the survival group and death group in total, and on the basis of each subgroup, were compared by Student's $t$ test. A receiver operating characteristic (ROC) curve with an area under the ROC curve (AUC) was used to analyze the performance of "initial CT" in predicting outcomes, and an optimal cutoff value with maximum sensitivity and specificity was obtained. Adjusted ROC curve was applied to exclude the influence of confounding factors of age and gender. $p$ value less than 0.05 (two-tailed) was considered statistically significant.

\section{Results}

\section{General information}

Epidemiologically, in our retrospective cohort, all 98 patients (mean age, $71.1 \pm 8.5$ years old; $66.3 \%$ are men) were local residents of Wuhan City; 2 patients acknowledged a history of contact with Wuhan's Huanan seafood market; 2 patients had familial cluster exposure; and 1 patient was a medical staff. There was no significant difference in age distribution between the death group and survival group $(72.7 \pm 8.9$ vs $69.7 \pm 8.0, p=0.074)$. The proportion of men was significantly higher in the death group than the survival group $(78.3 \% \mathrm{vs}$ $55.8 \%, p=0.019)$ (Table 1). In total, 67/98 (68.4\%) patients were having comorbidities and 46/98 (46.9\%) suffered from hypertension. In reviewed comorbidities, the proportion rate of chronic renal disease in the death group was statistically higher $(p=0.027)$ than that in the survival group. The median time interval of symptom onset to "initial CT" scan was 7 days (IQR, 3.75-10). There was no significant difference ( $p=$ 0.142 ) in the time interval of symptom onset and "initial CT" scan on admission between the two groups. The median hospital stay in the survival group was significantly longer than that in the death group ( 24 days vs 7.0 days, $p<0.001$ ).

\section{Clinical symptoms and treatments}

The most common clinical manifestations of patients in this study were fever (78/98 [79.6\%]) and cough (44/98 [44.9\%]). The proportion of dyspnea in the death group was significantly higher than that in the survival group $(p=0.007)$. There was no significant difference for other symptoms like fever, cough, chest tightness, weakness, and diarrhea.

All 98 patients received isolation and antiviral agents and oxygen therapy (including nasal oxygen breath and mask oxygen inhalation). Most patients (80\%) were empirically treated with antibiotics and glucocorticoids. Some patients (30\%) also received immunoglobulin treatment. In the death group, $19 / 46(41.3 \%)$ of patients required non-invasive mechanical ventilation, 14/46(30.4\%) patients required invasive mechanical ventilation, and 1 patient required extracorporeal membrane oxygenation (ECMO) treatment. In the survival group, 1 patient required non-invasive mechanical ventilation, and no patients received invasive mechanical ventilation or ECMO.

\section{Chest CT evaluation}

Of the 98 patients, 84 underwent chest CT scans before admission, and 14 underwent $\mathrm{CT}$ scans within $24 \mathrm{~h}$ after admission. According to the subgrouping rules mentioned earlier, in the death group, there were 24 patients in subgroup 1,16 patients in subgroup2, and 6 patients in subgroup3. In the survival group, there were 22 patients in subgroup1, 17 patients in subgroup2, and 13 patients in subgroup3.

GGO and consolidation are two main signs of COVID-19 lesions on chest $\mathrm{CT}$ images with or without vascular enlargement, interlobular septal thickening, air bronchogram sign, and air trapping, and the lesions were predominantly peripheral and subpleural. On CT images, 35 of the total 260 lobes were not involved in patients of the survival group, and 8 of the total 230 lobes were not involved in patients of the death group. A total of $53.2 \%$ (118/222) lobes involved in the death group and $32 \%$ (72/225) lobes involved in the survival group showed predominantly consolidation. Consolidations on CT images were more common in the death group than in the survival group $(p<0.001)$ (Figs. 2 and 3). Pleural effusion was found in CT images of 2 patients in the death group. No mediastinal lymphadenopathy was seen on chest $\mathrm{CT}$ images. 
Table 1 Demographic and clinical characteristics of patients in the death and survival groups

\begin{tabular}{|c|c|c|c|c|}
\hline Characteristics & $\begin{array}{l}\text { All patients } \\
(n=98)\end{array}$ & $\begin{array}{l}\text { Death } \\
(n=46)\end{array}$ & $\begin{array}{l}\text { Survival } \\
(n=52)\end{array}$ & $\begin{array}{l}p \\
\text { value* }\end{array}$ \\
\hline \multicolumn{5}{|l|}{ Age } \\
\hline Mean $\pm \mathrm{SD}$ (year) & $71.1 \pm 8.5$ & $72.7 \pm 8.9$ & $69.7 \pm 8.0$ & \multirow[t]{2}{*}{0.074} \\
\hline Range (year) & $60-95$ & $60-95$ & $61-92$ & \\
\hline \multicolumn{5}{|l|}{ Sex } \\
\hline Male & $65(66.3 \%)$ & $36(78.3 \%)$ & $29(55.8 \%)$ & \multirow[t]{2}{*}{0.019} \\
\hline Female & $33(33.7 \%)$ & $10(21.7 \%)$ & $23(44.2 \%)$ & \\
\hline \multicolumn{5}{|l|}{ History of exposure } \\
\hline Familial cluster & $2(2.0 \%)$ & 0 & $2(3.8 \%)$ & 0.179 \\
\hline Medical staff & $1(1.0 \%)$ & 0 & $1(1.9 \%)$ & 0.344 \\
\hline Huanan market & $2(2.0 \%)$ & 0 & $2(3.8 \%)$ & 0.179 \\
\hline \multicolumn{5}{|l|}{ Symptoms } \\
\hline Fever & $78(79.6 \%)$ & $35(76.1 \%)$ & $43(82.7 \%)$ & 0.418 \\
\hline Cough & $44(44.9 \%)$ & $25(54.3 \%)$ & $19(36.5 \%)$ & 0.077 \\
\hline Dyspnea & $17(17.3 \%)$ & $13(28.3 \%)$ & $4(7.7 \%)$ & 0.007 \\
\hline Chest tightness & $9(9.2 \%)$ & $7(15.2 \%)$ & $2(3.8 \%)$ & 0.052 \\
\hline Fatigue and poor appetite & $21(21.4 \%)$ & $11(23.9 \%)$ & $10(19.2 \%)$ & 0.573 \\
\hline Diarrhea & $11(11.2 \%)$ & $7(15.2 \%)$ & $4(7.7 \%)$ & 0.239 \\
\hline Rhinorrhea & & 0 & $1(1.9 \%)$ & 0.344 \\
\hline \multicolumn{5}{|l|}{ Comorbidities } \\
\hline Hypertension & $46(46.9 \%)$ & $21(45.7 \%)$ & $25(48.1 \%)$ & 0.810 \\
\hline Type 2 diabetes & $14(14.3 \%)$ & $5(10.9 \%)$ & $9(17.3 \%)$ & 0.363 \\
\hline Coronary heart disease & $15(15.3 \%)$ & $9(19.6 \%)$ & $6(11.5 \%)$ & 0.271 \\
\hline COPD & $7(7.1 \%)$ & $5(10.9 \%)$ & $2(3.8 \%)$ & 0.178 \\
\hline Postoperative lung cancer & $2(2.0 \%)$ & $1(2.2 \%)$ & $1(1.9 \%)$ & 0.930 \\
\hline Chronic renal disease & $10(10.2 \%)$ & $8(17.4 \%)$ & $2(3.8 \%)$ & 0.027 \\
\hline Chronic hepatitis & $2(2.0 \%)$ & $2(4.3 \%)$ & 0 & 0.129 \\
\hline None & $31(31.6 \%)$ & $13(28.3 \%)$ & $18(34.6 \%)$ & 0.500 \\
\hline $\begin{array}{l}\text { Symptom onset before CT, median (IQR) } \\
\text { (days) }\end{array}$ & $7(3.75-10)$ & $6(2.75-10)$ & $8(4-11)$ & 0.142 \\
\hline Hospital stay, median (IQR) (days) & $16.5(7.75-25)$ & $7(3-11)$ & $24(19-29)$ & $<0.001$ \\
\hline
\end{tabular}

*Between death group and survival group

$C O P D$, chronic obstructive pulmonary disease
The mean CT score was significantly higher in the death group than in the survival group ( $34.5 \pm 15.0$ vs $18.6 \pm 11.3$, $p<0.001)$. For subgroup1 and subgroup2, a higher mean CT score was found for the death group $(33.0 \pm 17.1 \mathrm{vs} 12.9 \pm 8.7$, $p<0.001 ; 38.8 \pm 12.3$ vs $24.3 \pm 11.9, p=0.002$, respectively) and no significant difference of CT score was identified with respect to subgroup3 $(28.8 \pm 11.5$ vs $20.7 \pm 10.4, p=0.144)$ (Fig. 4).

From ROC curve analysis, in subgroup1, CT score of 14.5 with a sensitivity of $83.3 \%$ and a specificity of $77.3 \%$ for the prediction of mortality was an optimal cutoff value, with an area under the curve (AUC) of 0.875 (95\% CI 0.778-0.972) and adjusted AUC of 0.881 (95\% CI 0.789-0.976). In subgroup2, CT score of 27.5 with a sensitivity of $87.5 \%$ and a specificity of $70.6 \%$ for the prediction of mortality was an optimal cutoff value, with an AUC of 0.814 (95\% CI 0.660 0.969 ) and adjusted AUC of 0.895 (95\% CI 0.677-0.977) (Fig. 5).

\section{Discussion}

In clinical practice, clinicians with isolation of protective clothing cannot auscultate and no frequent CT scans are performed for patients with COVID-19. Thus, using information of "initial CT" scan on admission to assess and 

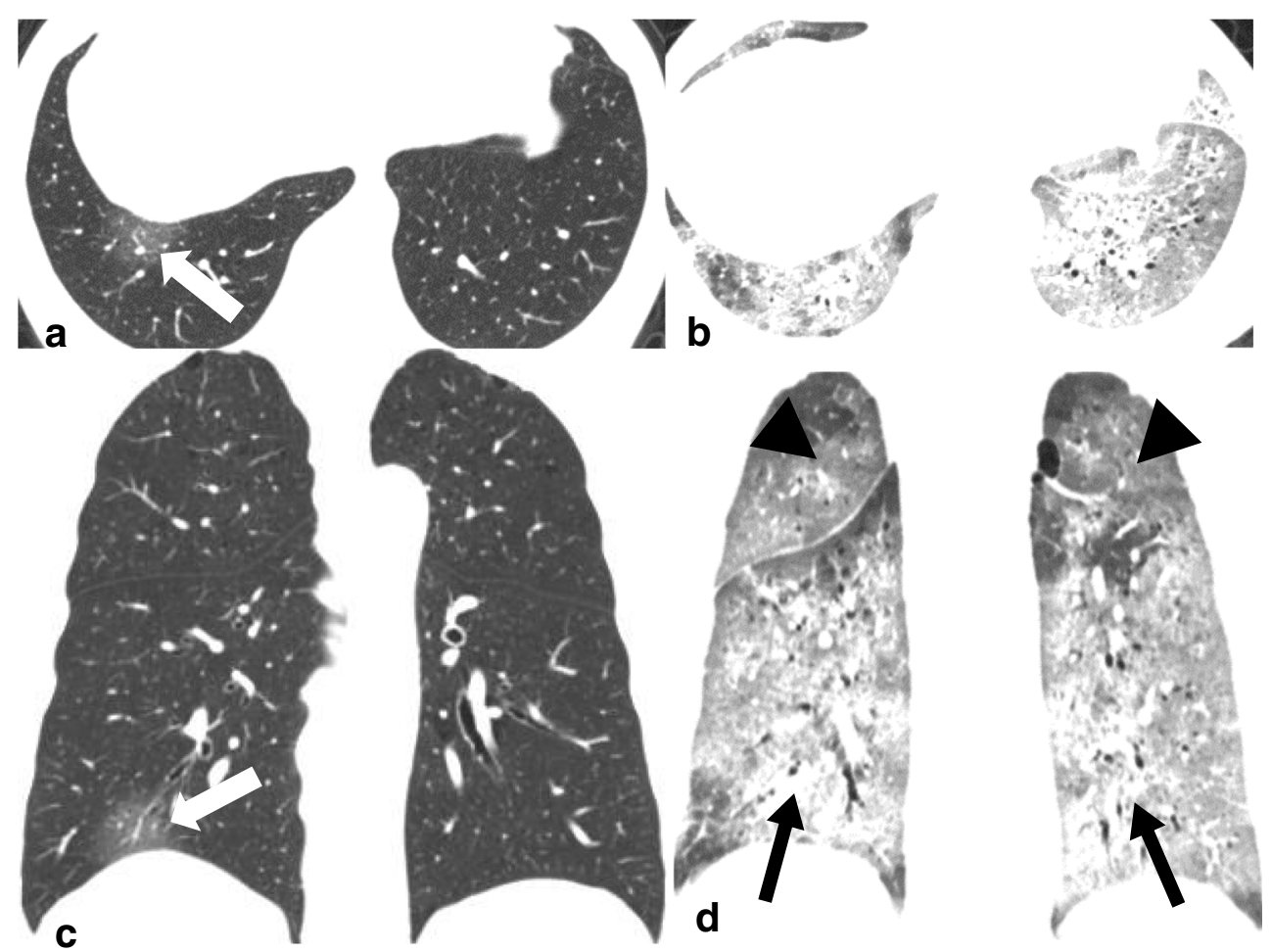

Fig. 2 A 61-year-old male with COVID-19 in the death group. a, c Transverse and coronal CT images with score of $2(1[1-25 \%] * 2$ [GGO]) showed GGO (white arrow) in the right lower lobe when the patient underwent CT scan on the same day with fever onsets. b, d Transverse and coronal CT images with score of 40 (calculated as 3 [50-75\% distribution in right upper lobe] $* 2$ [GGO, black arrow head]
+3 [50-75\% distribution in left upper lobe] $* 2$ [GGO, black arrow head] $+4[>75 \%$ distribution in right lower lobe] $* 3$ [consolidation, black arrow $]+4[>75 \%$ distribution in left lower lobe] $* 3$ [consolidation, black arrow $]+2$ [GGO, not showed $] * 2[25-50 \%$ distribution in right middle lobe]) showed lesions in whole lung lobes 9 days after onset (10 days before death) predict severity of the disease is useful and valuable for planning treatment strategies and evaluating prognosis. In this study, the CT score calculated from the "initial CT" scan can effectively predict mortality in older patients with COVID-19, showing a sensitivity of $83.3 \%$, a specificity of $77.3 \%$, and an adjusted AUC value of 0.881

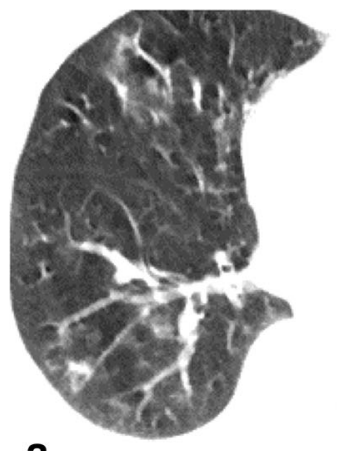

a

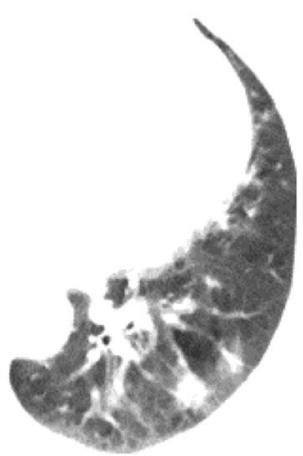

Fig. 3 A 66-year-old female with COVID-19 in the survival group who caught a fever on February 18, 2020, and admitted at the hospital on February 28, 2020. a On February 23, transverse CT with score of 10 showed GGO in the right middle lobe and consolidation in both lower (cutoff is 14.5), when the "initial CT" scans were done within 5 days after symptom onsets; the corresponding performance changes to a sensitivity of $87.5 \%$, a specificity of $70.6 \%$, and an adjusted AUC of 0.895 (cutoff is 27.5) when the time interval was 6 to 10 days between symptom onsets and CT scan.
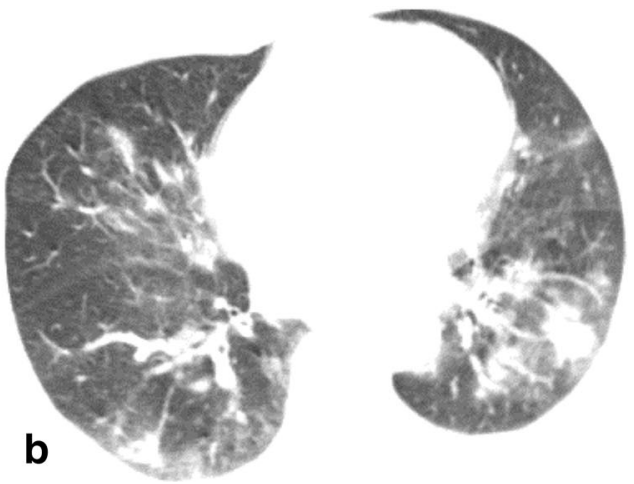

lobes. b On February 27, transverse CT with score of 24 showed increased consolidation, which was defined as "initial CT" according to the rules in this study 


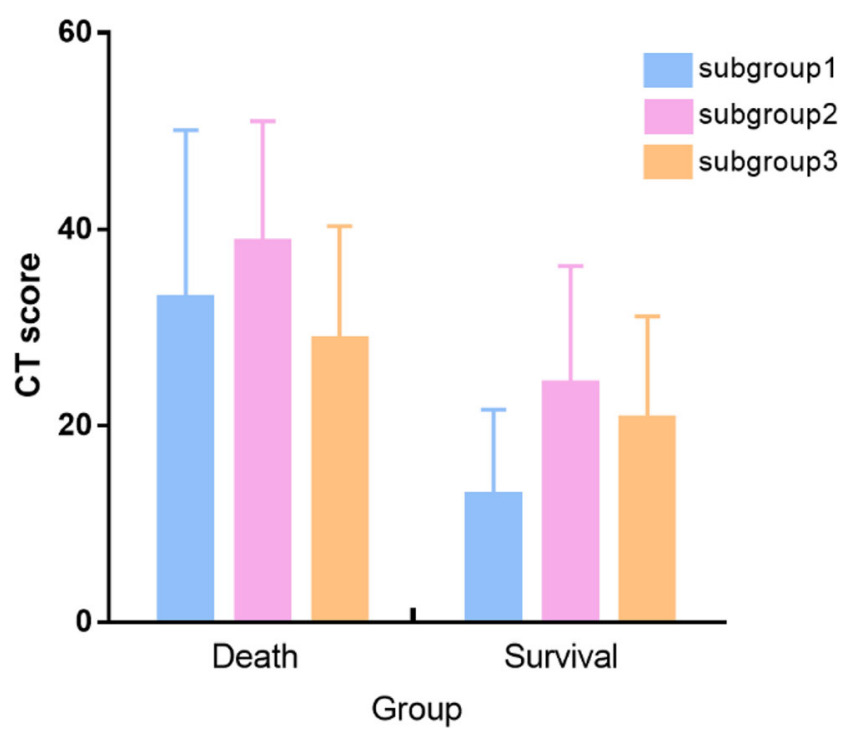

Fig. 4 CT scores of different subgroups in the death and survival groups. (In the survival or death group, patients were sub-grouped based on the interval time between symptom onset and the initial CT scan: subgroup1 (interval $\leq 5$ days after symptom onset), subgroup2 (interval between 6 and 10 days), and subgroup3 (interval $>10$ days)

From previous studies, it has been shown that older age, gender of male, and co-existing comorbidities might be risk factors for the poor prognosis of COVID-19 patients [4-6, 14]. In this single-center study on older patients, the proportion of men was significantly higher in the death group than in the survival group $(p=0.019)$, which was consistent with what was observed in a previous study [15]. However, it is important to notice that there was no significant difference in age distribution between the death group and survival group. In total, the proportion of severe symptom onsets (dyspnea) and co-existence with chronic renal disease was higher in the death group than in the survival group.

Two main CT signs of COVID-19 lesions are GGO and consolidation, which are similar to the $\mathrm{CT}$ features for SARS and MERS, and the lesions were predominantly peripheral and subpleural, consistent with previous studies [9, 10, 13, 14, 16, 17]. Consolidations were more common in the death group than in the survival group. These CT findings may correspond to viscous secretions seeping through the pulmonary alveoli described in the autopsy report [18] and suggest acute respiratory distress syndrome (ARDS). ARDS characterized by an acute, diffuse, inflammatory lung injury, leading to an increased alveolar capillary permeability, is a strong predictor of mortality, reflecting the severity of respiratory failures [19]. Our results supported the investigation that chest $\mathrm{CT}$ is possible to predict clinical course of ARDS [12, 20].
In this study, the overall $\mathrm{CT}$ score was based on the severity of air-space abnormality and its distribution. The mean CT score of chest CT images in the death group was significantly higher than that in the survival group. However, as previously reported [14], serial CT showed disease changes, and the most common pattern of evolution was initial progression to a peak level, followed by improvement. Our results showed that the "initial CT" score changes with respect to different time intervals between symptom onsets and CT scans. In subgroup1 and subgroup2, patients underwent CT scans within 10 days after symptom onsets, where the mean score of "initial CT" was statistically higher in the death group than in the survival group. This suggests that severe manifestation on CT at an early stage may indicate poor prognosis. In subgroup3, the time interval between symptom onsets and "initial CT" was longer than 10 days, where no significant difference was identified between the death group and survival group. According to Fig. 4, in either the death or survival group, CT score of chest CT in subgroup2 was higher (though not statistically significant) than that in subgroup 1 and subgroup3. This may indicate a progression course of 6-10 days after symptom onsets. However, this finding needs further evaluation.

This study had several limitations. First, we compared the scores of "initial CT" between the death and survival groups in terms of 3 subgroups, which does not account for individual variations. Second, due to the popularity of RT-PCR, many patients would not undergo CT scans for diagnosis before admission; thus, our study only included a relatively small number of cases who had initial CT scans acquired. Third, the CT scores were evaluated on the consensus of two readers rather than an independent reading, and there was a lack of assessment on the interobserver agreement. Fourth, because of the retrospective nature of the study, there is an inclusion bias, though we tried to avoid and such inclusion bias is inevitable, and our results should be carefully interpreted.

In summary, in this single-center study, we observed the prognosis implication of chest CT scores calculated by determining the extents of GGO and consolidation. We found that "initial CT" scores may be useful to stratify patients, which has a potentially important utility in the current global medical situation, especially for rational allocation of medical resources. However, a clear identification of the time interval between symptom onsets and "initial CT" is crucial. Further studies may include evaluation on a larger cohort and exploring the effects of this semi-quantitative method in monitoring disease progression and in assessing treatment responses. 

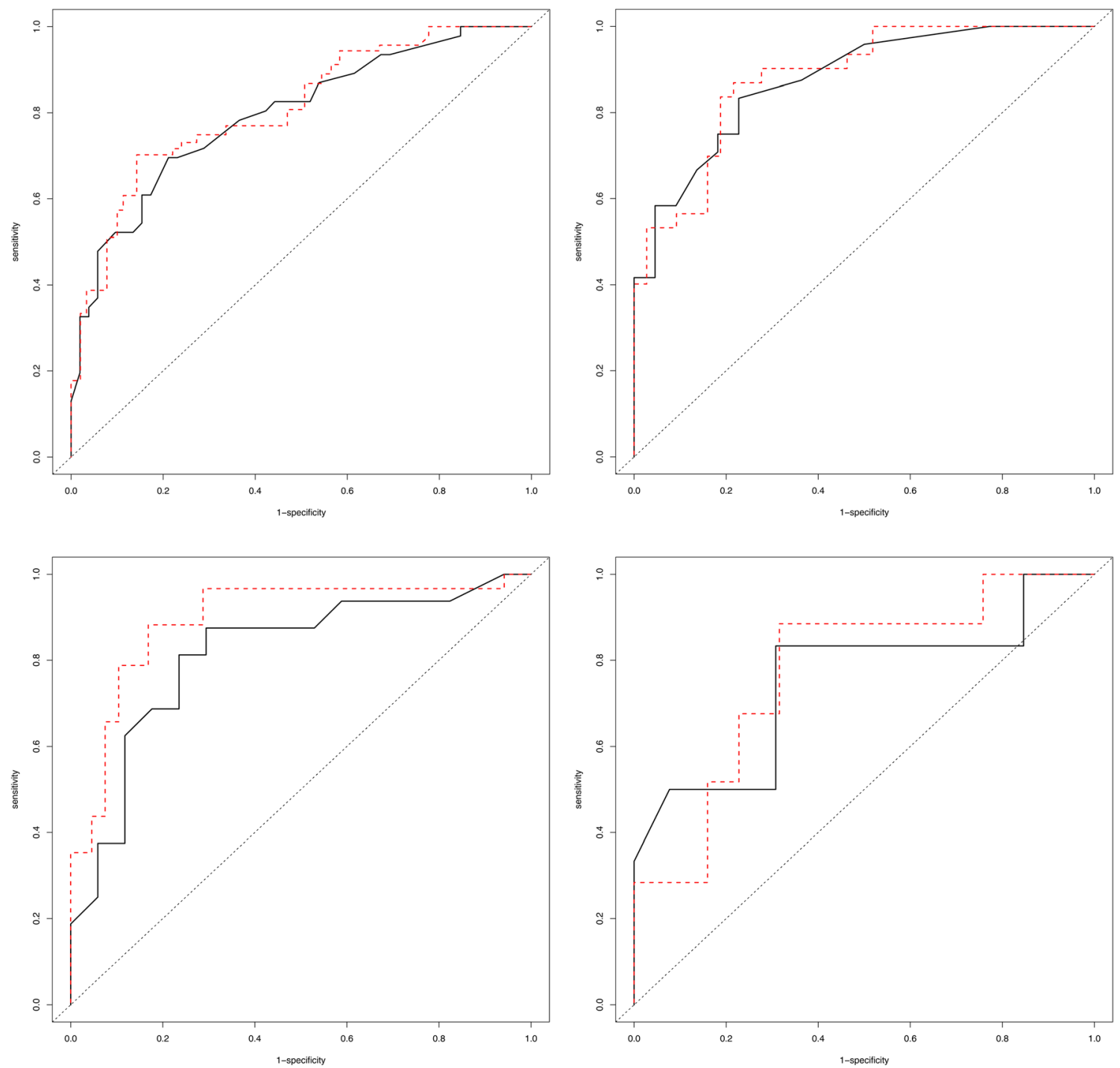

Fig. 5 ROC curve analysis of the CT scores including crude ROC curve and adjusted ROC curve after the adjustment for age and gender in the full cohort and in subgroup1, subgroup2, and subgroup3 for prediction of mortality

Funding information The authors state that this work has not received any funding.

\section{Compliance with ethical standards}

Guarantor The scientific guarantor of this publication is Prof. Liming Xia.

Conflict of interest The authors of this manuscript declare no relationships with any companies, whose products or services may be related to the subject matter of the article.

Statistics and biometry No complex statistical methods were necessary for this paper.

Informed consent Written informed consent was waived by the Institutional Review Board.

Ethical approval Institutional Review Board approval was obtained.
Study subjects or cohorts overlap No study subjects or cohorts have been previously reported before.

\section{Methodology}

- retrospective

- diagnostic or prognostic study

- performed at one institution

\section{References}

1. World Health Organization website (2020) Coronavirus disease (COVID-2019) situation reports. World Health Organization, Geneva Available via https://www.who.int/docs/default-source/ coronaviruse/situation-reports/20200421-sitrep-92-covid-19.pdf? sfvrsn=38e6b06d_4. Accessed 22 Apr 2020

2. World Health Organization website (2003) Summary of probable SARS cases with onset of illness from 1 November 2002 to 31 July 2003. World Health Organization, Geneva Available via 
http://www.who.int/csr/sars/country/table2004_04_21/en/. Accessed 15 Mar 2020

3. World Health Organization website (2019) Middle East respiratory syndrome coronavirus (MERS-CoV). World Health Organization, Geneva Available via http://www.who.int/emergencies/mers-cov/ en/. Accessed 15 Mar 2020

4. Yang XB, Yu Y, Xu JQ et al (2020) Clinical course and outcomes of critically ill patients with SARS-CoV-2 pneumonia in Wuhan, China: a single-centered, retrospective, observational study. Lancet Respir Med. https://doi.org/10.1016/s2213-2600(20)30079-5

5. Chen NS, Zhou M, Dong X et al (2020) Epidemiological and clinical characteristics of 99 cases of 2019 novel coronavirus pneumonia in Wuhan, China: a descriptive study. Lancet 395:507-513

6. Yang Y, Yang MH, Shen CG et al (2020) Evaluating the accuracy of different respiratory specimens in the laboratory diagnosis and monitoring the viral shedding of 2019-nCoV infections. medRxiv. https://doi.org/10.1101/2020.02.11.20021493

7. Wang YX, Liu WH, Yang M, Chen W (2020) The role of CT for Covid-19 patient's management remains poorly defined. Ann Transl Med. https://doi.org/10.21037/atm.2020.02.71

8. Kang Z, Li X, Zhou SC (2020) Recommendation of low-dose CT in the detection and management of COVID-2019. Eur Radiol. https:// doi.org/10.1007/s00330-020-06809-6

9. Ai T, Yang ZL, Hou HY et al (2020) Correlation of chest CT and RT-PCR testing in coronavirus disease 2019 (COVID-19) in China: a report of 1014 cases. Radiology. https://doi.org/10.1148/radiol. 2020200642:200642

10. Li Y, Xia LM (2020) Coronavirus disease 2019 (COVID-19): role of chest CT in diagnosis and management. AJR Am J Roentgenol. https://doi.org/10.2214/AJR.20.22954

11. National Health Commission of the People's Republic of China website (2020) Diagnosis and treatment of novel coronavirus infection (trial version 7). National Health Commission of the People's Republic of China, Beijing Available via http://www.nhc.gov.cn/ yzygj/s7652m/202003/a31191442e29474b98bfed5579d5af95. shtml. Accessed 15 Mar 2020
12. Ichikado K, Suga M, Muranaka $\mathrm{H}$ et al (2006) Prediction of prognosis for acute respiratory distress syndrome with thin-section CT: validation in 44 cases. Radiology 238:321-329

13. Chung M, Bernheim A, Mei XY et al (2020) CT imaging features of 2019 novel coronavirus (2019-nCoV). Radiology. https://doi. org/10.1148/radiol.2020200230

14. Shi HS, Han XY, Jiang NC et al (2020) Radiological findings from 81 patients with COVID-19 pneumonia in Wuhan, China: a descriptive study. Lancet Infect Dis. https://doi.org/10.1016/s14733099(20)30086-4

15. Huang CL, Wang YM, Li XW et al (2020) Clinical features of patients infected with 2019 novel coronavirus in Wuhan, China. Lancet. https://doi.org/10.1016/S0140-6736(20)30183-5

16. Pan YY, Guan HX, Zhou SC et al (2020) Initial CT findings and temporal changes in patients with the novel coronavirus pneumonia (2019-nCoV): a study of 63 patients in Wuhan, China. Eur Radiol. https://doi.org/10.1007/s00330-020-06731-x

17. $\mathrm{Xu} X, \mathrm{Yu} \mathrm{CC}, \mathrm{Qu} \mathrm{J}$ et al (2020) Imaging and clinical features of patients with 2019 novel coronavirus SARS-CoV-2. Eur J Nucl Med Mol Imaging. https://doi.org/10.1007/s00259-020-04735-9

18. Liu Q, Wang RS, Qu GQ et al (2020) Autopsy report of patients with novel coronavirus pneumonia. J Forensic Med. https://doi.org/ 10.12116/j.issn.1004-5619.2020.01.00 (In Chinese)

19. Fan E, Brodie D, Slutsky AS (2018) Acute respiratory distress syndrome: advances in diagnosis and treatment. JAMA 319:698710

20. Nishiyama A, Kawata N, Yokota $\mathrm{H}$ et al (2020) A predictive factor for patients with acute respiratory distress syndrome: CT lung volumetry of the well-aerated region as an automated method. Eur J Radiol. https://doi.org/10.1016/j.ejrad.2019.108748

Publisher's note Springer Nature remains neutral with regard to jurisdictional claims in published maps and institutional affiliations. 